The band gap scaling laws in group IV nanotubes

This content has been downloaded from IOPscience. Please scroll down to see the full text.

Download details:

IP Address: 128.41.35.98

This content was downloaded on 31/01/2017 at 13:12

Manuscript version: Accepted Manuscript

Wang et al

To cite this article before publication: Wang et al, 2017, Nanotechnology, at press:

http://dx.doi.org/10.1088/1361-6528/aa5b3e

This Accepted Manuscript is copyright Copyright 2017 IOP Publishing Ltd

During the embargo period (the 12 month period from the publication of the Version of Record of this article), the Accepted Manuscript is fully protected by copyright and cannot be reused or reposted elsewhere.

As the Version of Record of this article is going to be / has been published on a subscription basis, this Accepted Manuscript is available for reuse under a CC BY-NC-ND 3.0 licence after a 12 month embargo period.

After the embargo period, everyone is permitted to use all or part of the original content in this article for non-commercial purposes, provided that they adhere to all the terms of the licence https://creativecommons.org/licences/by-nc-nd/3.0

Although reasonable endeavours have been taken to obtain all necessary permissions from third parties to include their copyrighted content within this article, their full citation and copyright line may not be present in this Accepted Manuscript version. Before using any content from this article, please refer to the Version of Record on IOPscience once published for full citation and copyright details, as permissions will likely be required. All third party content is fully copyright protected, unless specifically stated otherwise in the figure caption in the Version of Record.

When available, you can view the Version of Record for this article at: http://iopscience.iop.org/article/10.1088/1361-6528/aa5b3e 


\title{
The band gap scaling laws in group IV nanotubes
}

\author{
Chongze Wang ${ }^{1,2}$, Xiaonan $\mathrm{Fu}^{3}$, Yangyang Guo ${ }^{1}$, Zhengxiao Guo ${ }^{5}$, Congxin $\mathrm{Xia}^{4, \dagger}, \mathrm{Yu} \mathrm{Jia}^{1,2^{*}}$ \\ ${ }^{1}$ International Laboratory for Quantum Functional Materials of Henan, and School of Physics \\ and Engineering, Zhengzhou University, Zhengzhou 450001, China \\ ${ }^{2}$ Key Laboratory for Special Functional Materials of Ministry of Education, Henan University, \\ Kaifeng 475004, China \\ ${ }^{3}$ College of Science, Henan University of Technology, Zhengzhou 450001, China \\ ${ }^{4}$ School of Physics and Materials Science, Henan Normal University, Xinxiang 453007, China \\ ${ }^{5}$ Department of Chemistry, University College London, London WC1E 6BT, United Kingdom
}

\begin{abstract}
By using the first-principles calculations, the band gap properties of nanotubes formed by group IV elements have been investigated systemically. Our results reveal that for armchair nanotubes, the energy gaps at $K$ points in Brillouin zone decrease as $1 / r$ scaling law with the radii $(r)$ increasing, while they are scaled by $-1 / r^{2}+\mathrm{C}$ at $\Gamma$ points, here, $\mathrm{C}$ is a constant. Further studies show that such scaling law of $\mathrm{K}$ point is independent of both the chiral vector and the type of elements. Therefore, the band gaps of nanotubes for a given radius can be determined by these scaling laws easily. Interestingly, we also predict the existence of indirect band gap for both germanium and tin nanotubes. Our new findings provide an efficient way to determine the band gaps of group IV element nanotubes by knowing the radii, as well as facilitate the design of functional nano-devices.

*Corresponding author: jiayu@zzu.edu.cn; xiacongxin@htu.edu.cn
\end{abstract}




\section{Introduction}

Very recently, the new two-dimensional (2D) monolayer of tin has been experimentally realized via epitaxial growth method [1]. That is to say, since we obtain graphene, we have almost realized all the 2D monolayers in group IV successively by various approaches expecting for metal lead element [2-6]. Like graphene, the other group IV monolayers, namely, silicene, germanene, and stanene, also behave like massless Dirac fermions properties, which give rise to ultimate high charge carrier mobility. Also, these 2D monolayers are promising to support 2D quantum spin Hall states and provide enhanced thermoelectricity, topological superconductivity and the quantum anomalous Hall effect, and so on [7-11].

Similarly, the one dimensional nanotubes based on those group IV elements have also attracted increasingly interests with the achievement of these two-dimensional monolayers. In fact, carbon nanotubes (CNTs) have aroused considerable scientific interests due to their novel electronic properties and applications in many fields even earlier than graphene, such as CNT canning probe, CNT field effect transistors, intermolecular metal-semiconductor heterojunctions diodes, single electron tunneling devices, CNT field emitters, CNT sensors composites [12-18]. As we known, one intriguing property of the carbon nanotubes is that they can exhibit metallic or semiconductor properties depending on their chiral vectors. For armchair carbon nanotubes, they always exhibit metallic properties, and there is a metallic semiconducting oscillation with a period of 3 for zigzag carbon nanotubes. For silicon nanotubes, due to their unique gear-like structures caused by partial $s p^{3}$ hybridization, previous studies reveal that the armchair nanotubes exhibit semiconductor properties with decreasing band gaps as radii of nanotubes increase. On the other hand, band gaps also oscillate with a period of 3 for the zigzag silicon nanotubes. [19-21]. 
Structurally, the single wall nanotubes (SWNTs) can be considered as quasi-onedimensional tubular structures rolled up from 2D monolayer sheets, and chiral vector $(m, n)$ has been introduced to describe the circumferential of nanotubes. Therefore, electronic properties of carbon SWNTs can be deduced from that of $2 \mathrm{D}$ graphene by Brillouin zone (BZ) folding scheme [22-24]. Following this assumption, one can conclude that the nanotubes in group IV elements will exhibit similar electronic properties. Since stanene was experimentally realized recently, there are also various theoretical investigation about the electronic properties of tin monolayer, as well as the nanoribbons [25-27]. However, tin nanotubes have not been discussed yet, and the electronic properties of those nanotubes are still elusive. Therefore, in this paper, by using first-principles calculations, we investigate the generality of the band gap properties of a large range of carbon-, silicon-, germanium- and tin-based SWNTs. Armchair nanotubes (m, m) and zigzag nanotubes $(\mathrm{m}, 0)$ are constructed and their band structures are studied systematically. Our results show that band gaps of these nanotubes obey two universal scaling laws at $\mathrm{K}(k=2 \pi / 3 \mathrm{a})$ and $\Gamma$ points, respectively. Based on these two scale laws, we present a simple way to determine the band gaps of nanotubes of IV group elements only with their radii.

\section{Computational method}

In the implementation of our first-principles calculations, we use the Vienna abinitio simulation package (VASP) to solve the Kohn-Sham equations within the framework of density function theory [28-30]. The Perdew-Burke-Ernzerhof [31] generalized-gradient approximation is adopted in describing the exchange-correlation interaction. The Brillouin zone sampling using Monkhorst-Pack scheme is carried out with $1 \times 1 \times 13$ meshes for armchair nanotubes and $1 \times 1 \times 11$ meshes for zigzag nanotubes 
[32]. The plane-wave cutoff energy is set to $800 \mathrm{eV}$ for carbon and $500 \mathrm{eV}$ for silicon, germanium and tin, respectively. The Gaussian smearing factor is set to be $0.05 \mathrm{eV}$, and the vacuum layer larger than $18 \AA$ is employed to ensure the decoupling of repeated nanotubes in all calculations. In order to optimize the geometric structures, we relax all the atoms in the systems until the atomic force on each atom is less than $0.02 \mathrm{eV} / \AA$, we also have checked the accuracy with the atomic force on each atom less than $0.01 \mathrm{eV} / \AA$ and we find the present results can well describe the band gap properties of group IV nanotubes. Moreover, we have checked our results by including the vdW corrections within the method of Tkatchenko and Scheffler [33], and the results show that the influence of vdW can be ignored when discussing the band gaps of single-wall nanotubes. For the discussion of the thermodynamic stability, the phonon spectrums are calculated by using the package phonopy [34] with the forces calculated with VASP code.

\section{Results and discussion}

Because of the buckling structures of silicene, germanene and stanene, their corresponding nanotubes have gear-like structures, quite different from carbon nanotubes. The gear-like structures of nanotubes are presented in Fig .1 (a), in which the cross-section and side views are given for tin armchair $(8,8)$ and zigzag $(10,0)$ nanotubes, respectively. Due to the gear-like structures, there are two classes of atoms with small and large distance to the central axis, which are illustrated using different colors in Fig. 1 (a). In the following discussion, the smaller one is employed as the radius of the nanotube, denoted as $r$. Actually, choosing the bigger one or the average value as the radius of nanotube has no influence on the results discussed below.

First, we begin with the discussions of the thermodynamic stability of the group 
IV nanotubes. Of course, there are no doubts about the thermodynamic stability of carbon nanotubes for there are many applications in both electronic and optical fields. It has been reported that silicon nanotubes could be stable at about $500 \mathrm{~K}$ from the molecular dynamics simulations [35]. In order to investigate the thermodynamic stability of carbon, silicon, germanium, and tin nanotubes systematically, here we have performed the calculations of the phonon spectrum for carbon, silicon, germanium, and tin nanotubes with different radii and chiral vectors. As examples, here we only present the phonon spectrums of armchair $(8,8)$, and zigzag $(10,0)$ nanotubes respectively, as shown in Fig .1 (b) (c). From these results, we see that there are no imaginary frequencies throughout the entire Brillouin zone, indicating the stability of nanotubes formed by carbon, silicon, germanium, and tin, respectively. 


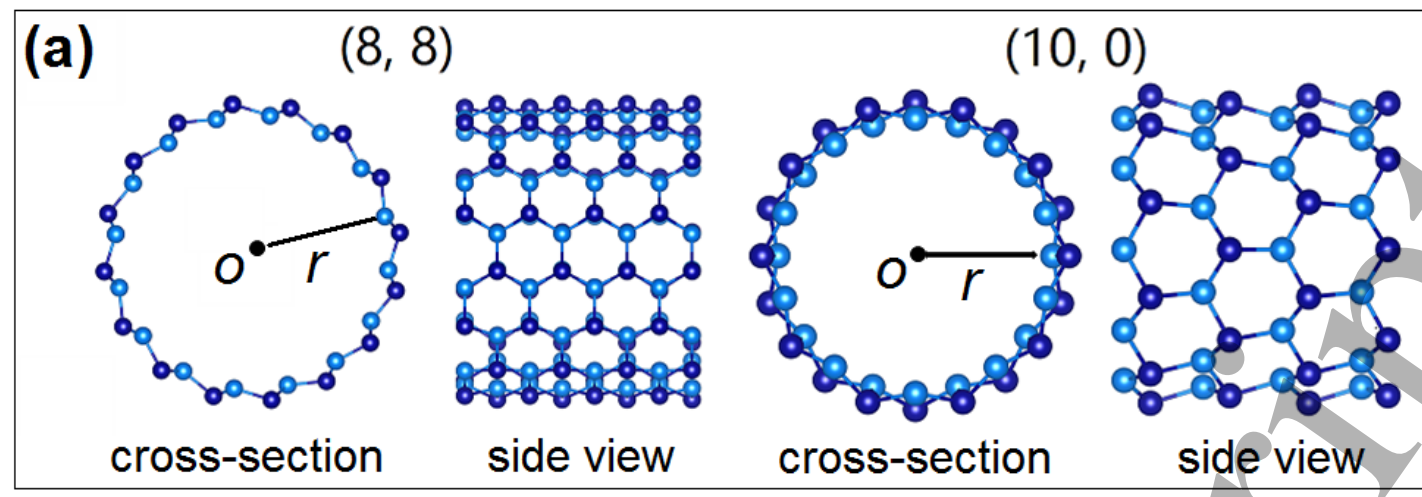

(b)
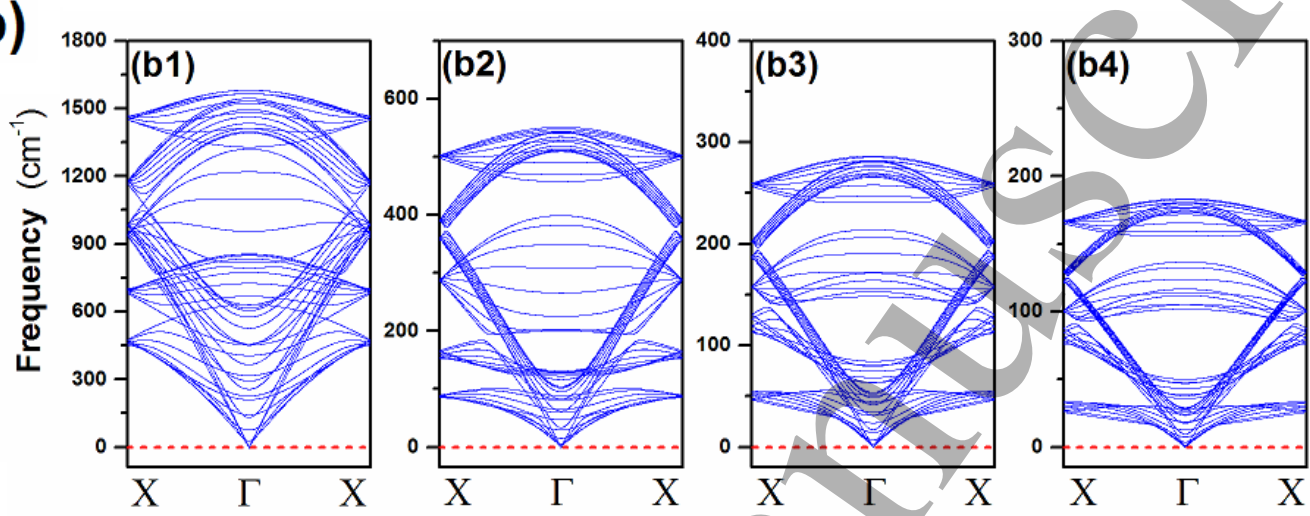

(c)
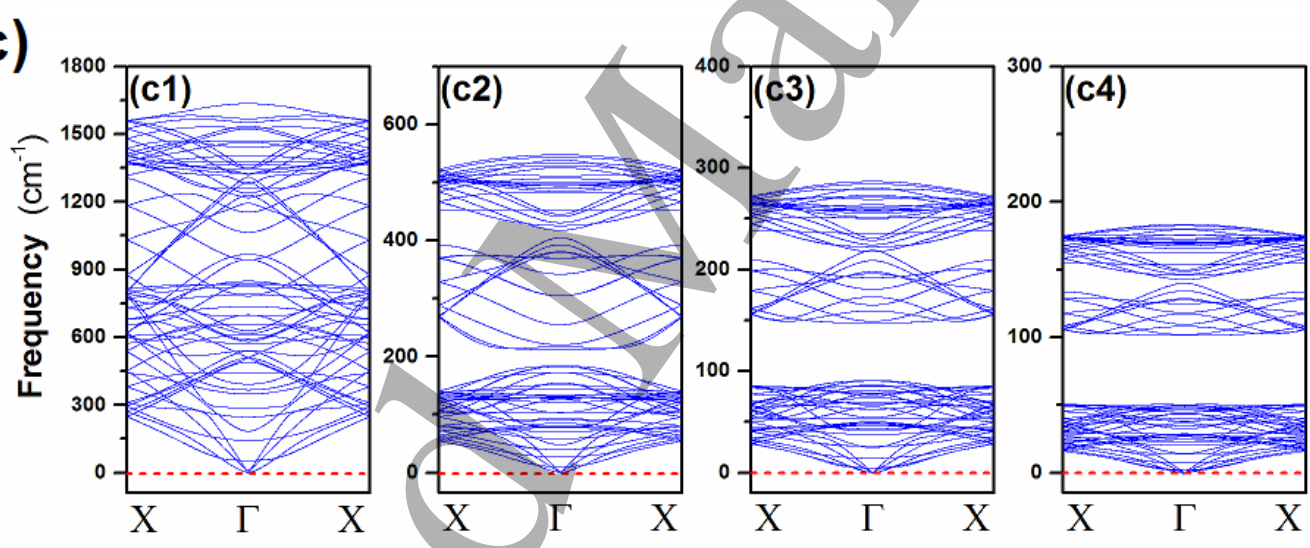

Fig. 1. (a) Structures of tin armchair $(8,8)$ and zigzag $(10,0)$ nanotubes. The two classes of atoms in gear-like structures are distinguished by color. Calculated phono spectrums of armchair (8, 8) (b) and zigzag (10, 0) (c) nanotubes formed by carbon (b1, c1), silicon (b2, c2), germanium (b3, c3), and tin (b4, c4), respectively.

In Fig. 2, we present the band structures of armchair nanotubes with chiral vectors of $(3,3),(8,8)$ and $(13,13)$ for carbon, silicon, germanium, and tin, respectively. There are two high symmetry points in the 1D Brillouin zone of nanotube, namely $\Gamma$ point with $k=0$ and $\mathrm{X}$ point with $k= \pm \pi / a$, where $a$ is the lattice constant of nanotubes. There 
is another important point between $\Gamma$ and $\mathrm{X}$ for armchair nanotubes, namely $\mathrm{K}(k=$ $2 \pi / 3 a)$. According to BZ folding scheme, the Dirac cones located at $(2 \pi / 3 a, 2 \pi / 3 a)$ points in two dimensional BZ of corresponding 2D monolayers is folded into $\mathrm{K}(k=$ $2 \pi / 3 a$ ) in 1D Brillouin zone [22-24]. Therefore, there are two special points which have much influence on the magnitude of band gaps for armchair nanotubes, namely, $\Gamma$ and K points. For carbon nanotubes, as shown Fig. 2(a), we see that they exhibit metallic properties with zero gaps and the Dirac cones are located at $\mathrm{K}$ point, which is in agreement with previous theoretical studies [22-24]. For silicon nanotubes, as shown in Fig. 2(b), there are energy gaps at $\mathrm{K}$ points, and here we denoted as $g_{\mathrm{K}}$, namely the difference between the conduction band minimum $\left(\mathrm{CBM}_{\mathrm{k}}\right)$ and the valence band maximum $\left(\mathrm{VBM}_{\mathrm{k}}\right)$ at $\mathrm{K}$ point. With the increasing radii $r$ of silicon nanotubes, the $g_{\mathrm{K}}$ will decreases dramatically. Compared with carbon nanotubes, opening a gap $g_{\mathrm{K}}$ is attribute to the lower symmetrical gear-like structures of silicon nanotubes [22]. For the germanium and tin armchair nanotubes, the variation of $g_{\mathrm{K}}$ as a function of $r$ is the same as that of silicon nanotubes. However, the band structures of germanium and tin armchair nanotubes at $\Gamma$ are totally different from those of both carbon and silicon. From Fig. 2(c) and (d), the germanium $(3,3)$ and tin $(3,3)$ nanotubes have a direct band gap located at $\Gamma$ point where it is much smaller than that of $\mathrm{K}$ point, denoted as $g_{\Gamma}$, representing the difference between the $\mathrm{CBM}_{\Gamma}$ and the $\mathrm{VBM}_{\Gamma}$ at $\Gamma$ point. With increasing $r$, the $g_{\Gamma}$ becomes larger while the $g_{\mathrm{K}}$ smaller. For the $(8,8)$ and $(13,13)$ nanotubes of germanium and tin, $g_{\Gamma}$ are larger than $g_{\mathrm{K}}$, and $g_{\mathrm{K}}$ become the real band gaps of the nanotubes. Taken together, it is clear from Fig. 2 that, for armchair nanotubes formed by group IV elements, $g_{\Gamma}$ and $g_{\mathrm{K}}$ exhibit different variation trends as the functions of $r$, which are important to determine the band gaps of the nanotubes. 

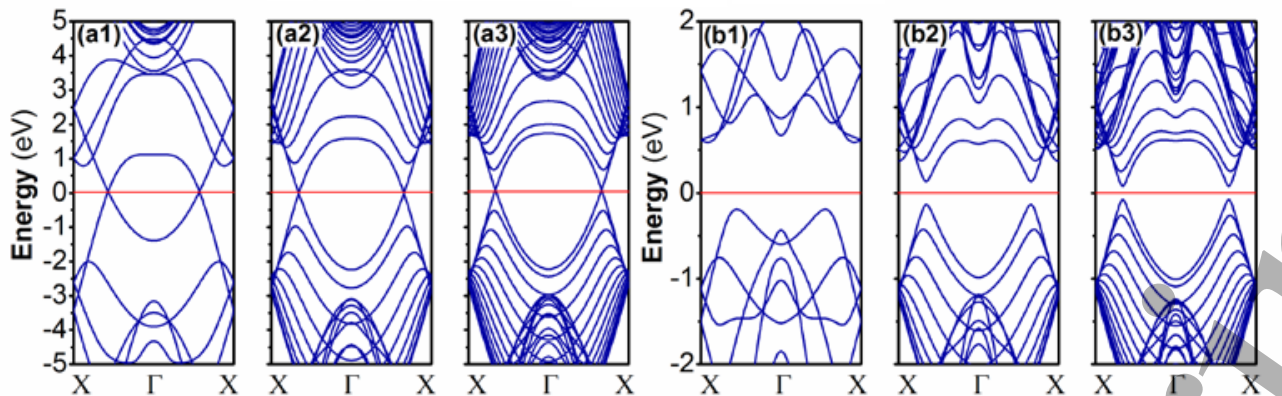

(a)

(b)

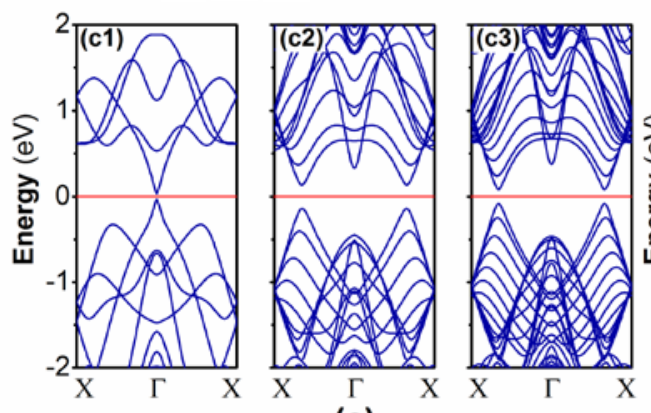

(c)
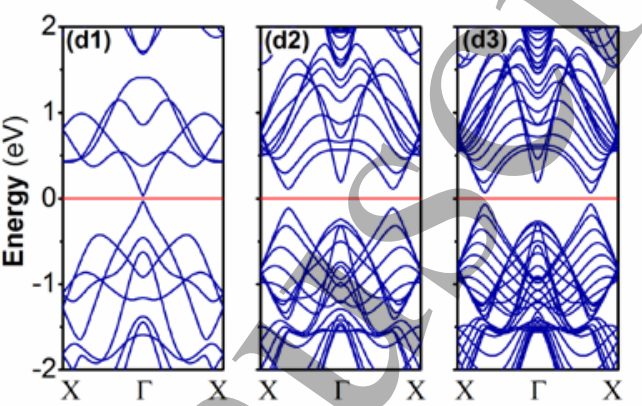

(d)

Fig. 2. Band structures of armchair $(3,3)(1),(8,8)(2)$, and $(13,13)(3)$ nanotubes for carbon

(a) silicon (b) germanium (c) and tin (d), respectively.

Motivated by above calculations, we investigate the changes of $g_{\mathrm{K}}$ and $g_{\Gamma}$ of armchair nanotubes as the functions of $r$. In Fig. 3(a), we present the changes of the CBMк and the $\mathrm{VBM}_{\mathrm{K}}$ of armchair nanotubes for silicon, germanium and tin as the functions of $r$, in which the Fermi levels are set to zero. By fitting our calculated results, we find that the $\mathrm{CBM}_{\mathrm{K}}$ obeys a $1 / r$ scaling law while $\mathrm{VBM}_{\mathrm{K}}$ a $-1 / r$ scaling law. Moreover, the changes of the $\mathrm{CBM}_{\mathrm{K}}$ and the $\mathrm{VBM}_{\mathrm{K}}$ are symmetric regarding to the Fermi levels. Very interestingly, we reveal that the scaling laws are independent of the type of the constitute elements.

To validate the universality of such scaling laws of the СВМк and the $\mathrm{VBM}_{\mathrm{K}}$ for armchair nanotubes with different chiral vectors, we also calculate the CBM and the VBM of zigzag $(\mathrm{m}, 0)$ nanotubes as the functions of $r$. According to the BZ folding scheme, the K point in 1D Brillouin zone of armchair nanotubes is corresponding to $\Gamma$ 
point in that of zigzag nanotubes when $\mathrm{m}=3 \mathrm{q}$ ( $\mathrm{q}$ is an integer) [22-24]. The calculated results of the CBM and the VBM for zigzag nanotubes are plotted in Fig. 3(b). Obviously, the CBM and VBM oscillates with $m$ in a period of 3 for silicon, germanium, and tin zigzag nanotubes. When $\mathrm{m}=3 \mathrm{q}$ ( $q$ is an integer), both the CBM and VBM of zigzag nanotubes also obey the same scaling laws (the functions of $r$ ) as those of armchair nanotubes. When $\mathrm{m} \neq 3 q$, both the CBM and the VBM have small shifts above or below $\pm 1 / r$, and the reason have been discussed previously [22-24]. Furtherrmore, we reveal that the scaling laws are also independent of the chiral vectors. This is to say, if the band gaps are determined by the CBM and the VBM at $\widehat{K}$ points, the values of the band gaps for both armchair and zigzag nanotubes can be obtained by only knowing the radii $r$. Here we have noticed that the previous studies of the silicon nanotubes could not give a consistent result of band gaps of armehair and zigzag nanotubes [21].

Now, we turn to study the changes of the $\mathrm{CBM}_{\Gamma}$ and $\mathrm{VBM}_{\Gamma}$ of armchair nanotubes as the functions of $r$. Fig. 3(c) gives the changes of the $\mathrm{CBM}_{\Gamma}$ and $\mathrm{VBM}_{\Gamma}$ with $r$ for carbon, silicon, germanium and tin nanotubes, respectively. By fitting the calculated results, we find that there are three features for the changes of the $\mathrm{CBM}_{\Gamma}$ and $\mathrm{VBM}_{\Gamma}$ with increasing $r$. Firstly, the values of the $\mathrm{CBM}_{\Gamma}$ increase as $-1 / r^{2}+\mathrm{C}_{1}$ while the $\mathrm{VBM}_{\Gamma}$ decrease as $1 / r^{2}+C_{2}$ scaling laws, where $C_{1}$ and $C_{2}$ are constants. Here, for germanium and tin nanotubes, the constants $\mathrm{C}_{1}$ and $\mathrm{C}_{2}$ are the values of the $\mathrm{CBM}_{\Gamma}$ and $\mathrm{VBM}_{\Gamma}$ of the corresponding 2D monolayers. Secondly, for carbon and silicon armchair nanotubes, there are no crossovers between the $\mathrm{CBM}_{\Gamma}$ and the $\mathrm{VBM}_{\Gamma}$ at Fermi levels. However, for germanium and tin armchair nanotubes, there are crossovers between the $\mathrm{CBM}_{\Gamma}$ and $\mathrm{VBM}_{\Gamma}$ at Fermi levels for a given $r$, namely $(3,3)$ nanotubes for both germanium and tin. Thirdly, the changes of the $\mathrm{CBM}_{\Gamma}$ and $\mathrm{VBM}_{\Gamma}$ as function of $r$ are asymmetric 
regarding to the Fermi levels since it is obviously that the absolute values of the $\mathrm{CBM}_{\Gamma}$ and the $\mathrm{VBM}_{\Gamma}$ are not equal when radius of nanotubes becomes infinite.
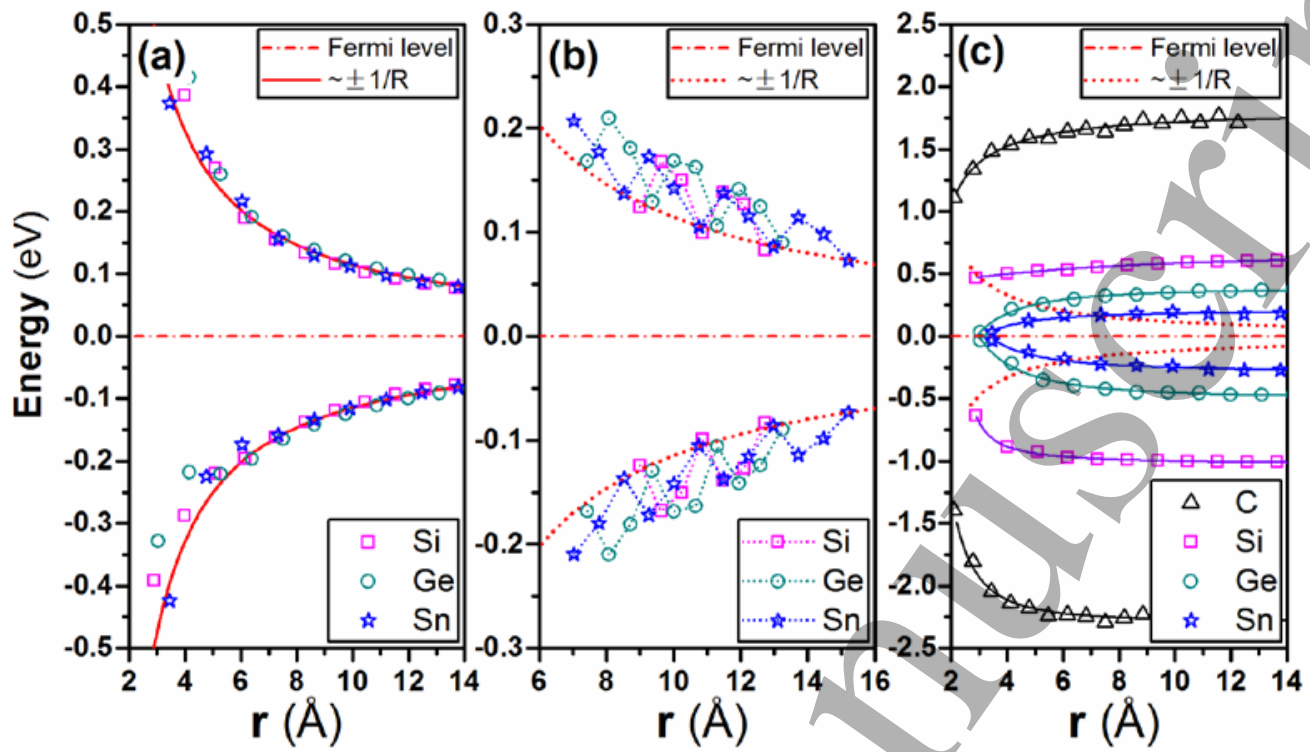

Fig. 3 (a) The calculated values of the $\mathrm{CBM}_{K}$ and $\mathrm{VBM}_{\mathrm{K}}$ of armchair nanotubes formed by group IV elements as the functions of $r$. The red lines in (a) present the scaling law of $\pm 1 / r$, and the dash dot line presents the Fermi levels. (b) The calculated values of the CBM and VBM of zigzag nanotubes for silicon, germanium and tin, respectively. (c) The values of the $\mathrm{CBM}_{\Gamma}$ and $\mathrm{VBM}_{\Gamma}$ of armchair nanotubes versus radii of carbon, silicon, germanium and tin respectively. The dash dot line present Fermi level in both (c) and (d).

Based on our above findings, we can determine the band gaps of various nanotubes formed by group IV elements for a given radius. From Fig. 3(c), for carbon and silicon nanotubes, the $\mathrm{CBM}_{K}$ is always smaller than the $\mathrm{CBM}_{\Gamma}$ whereas the $\mathrm{VBM}_{K}$ is larger than $\mathrm{VBM} г$, and therefore the band gaps of carbon and silicon nanotubes are only determined by $g_{\mathrm{K}}$, as we can see from Fig. 4(a) and (b). For germanium and tin nanotubes, when $r$ is small, the $\mathrm{CBM}_{\Gamma}$ is smaller than the $\mathrm{CBM}_{\kappa}$ and the $\mathrm{VBM}_{\Gamma}$ is larger 
than the $\mathrm{CBM}_{\kappa}$, the band gaps are determined by $g_{\Gamma}$. When $r$ is larger than $\sim 6 \AA$, the СBMк is smaller than the $\mathrm{CBM}_{г}$ and the $\mathrm{VBM}_{\kappa}$ is larger than the $\mathrm{VBM}_{г}$, so the band gaps of nanotubes are determined by $g_{\mathrm{K}}$. This is to say, for germanium and tin nanotubes, as $r$ increase, band gaps will increase firstly and then decrease, as shown in Fig. 4(c) and (d). However, for carbon nanotubes without the gear-like structures, $g_{\mathrm{K}}$ always equals to zero, and we regard it as an exception. Band gap properties of zigzag nanotubes with small radii for carbon have been explained by hybridization of the $\pi$ and $\sigma$ states [36-38] as well as the silicon nanotubes [21].
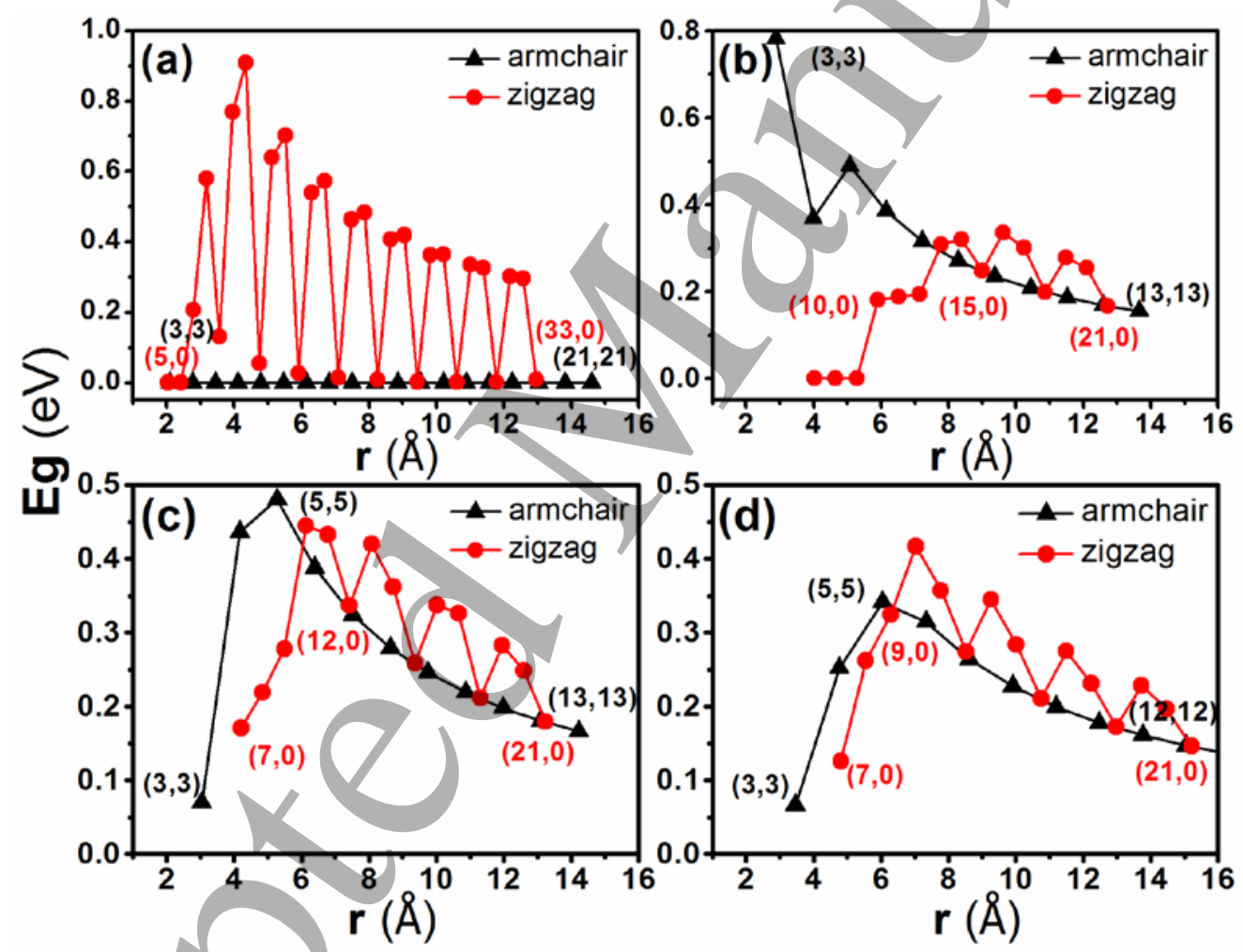

Fig. 4. Band gaps of nanotubes of (a) carbon, (b) silicon, (c) germanium and (d) tin with different chiral vectors and various radii, respectively. The red and black lines show the results of zigzag and armchair nanotubes, respectively. The chiral vectors of nanotubes is signed for some representative nanotubes.

It should be noticed that, for germanium $(4,4),(5,5)$ and tin $(5,5)$ nanotubes 
which their radii are close to $\sim 6 \AA$, the $\mathrm{CBM}_{\Gamma}$ is smaller than $\mathrm{CBM}_{K}$, but the $\mathrm{VBM}_{K}$ is larger than $\mathrm{VBM} г$, therefore the band gaps of nanotubes are determined by both the $\mathrm{CBM}_{\Gamma}$ at $\Gamma$ points and the $\mathrm{VBM}_{K}$ at $\mathrm{K}$ points. This results originated from that the changes of the $\mathrm{CBM}_{K}$ and $\mathrm{VBM}_{K}$ are symmetric and the $\mathrm{CBM}_{\Gamma}$ and $\mathrm{VBM}_{\Gamma}$ are asymmetric regarding to the Fermi levels. In Fig. 5(a) and (b), we have plotted the band structures of germanium $(5,5)$ and tin $(5,5)$ nanotubes, respectively. It is clear that for both the armchair nanotubes, the VBM located at K and CBM/located at $\Gamma$, resulting the indirect band gap properties. This is the first prediction about the indirect nanotubes for germanium and tin. Since the DFT-GGA calculations always underestimate the band gaps, we also do calculations by using the hybrid density functional HSE06 [39, 40], the results are present as inserts in Fig.5(a) and (b) respectively. Our calculations further verify there exist indirect band gap nanotubes.

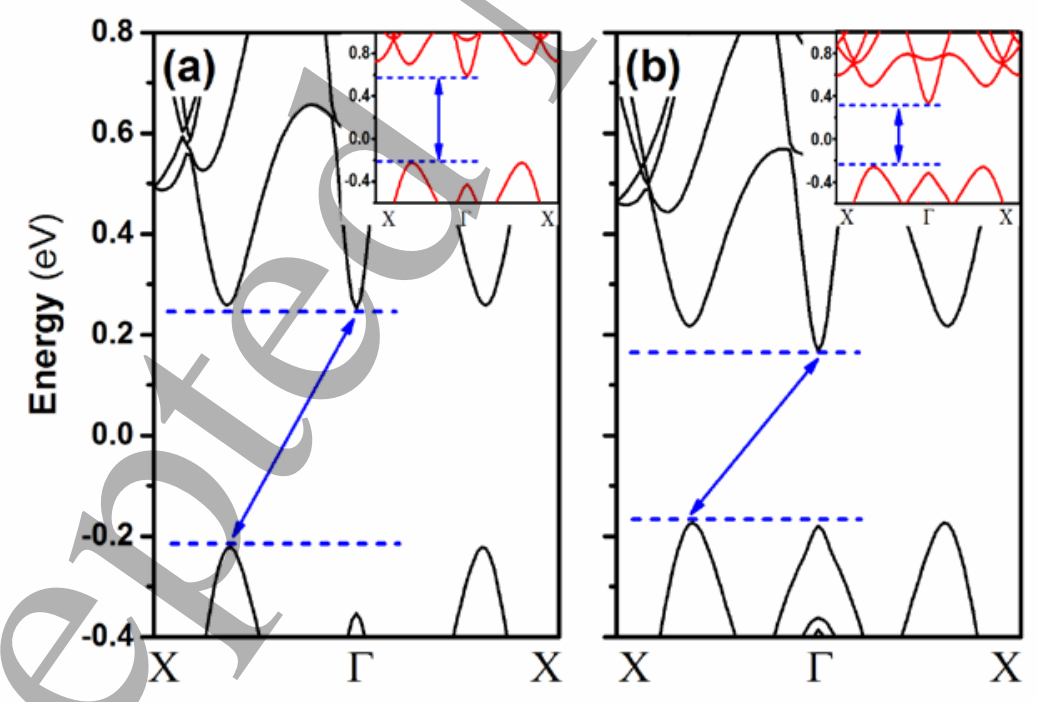

Fig. 5. Band structures of armchair nanotubes of (a) Ge (5, 5) and (b) Sn (5, 5), respectively.

The corresponding results of HSE06 calculations are shown as inserts separately. 


\section{Conclusion}

In summary, using the first-principles calculations, we have discussed the band gap properties for nanotubes formed by group IV elements. We have revealed that the energy gap $g_{\mathrm{K}}$ obeys a symmetric $1 / r$ scaling law, while $g_{\Gamma}$ obeys an asymmetric $-1 / r^{2}+C$. We also have revealed that the scaling law of $g_{\mathrm{K}}$ is independent of both the chiral vector and the type of elements. Based on these scaling laws, the band gaps of IV element nanotubes can be easily determined by the given values of radii. Interestingly, we first predicted that there exist the indirect band gap nanotubes for germanium and tin which may have potential applications in nanodevices.

\section{Acknowledgments}

This work is supported by the National Basic Research Program of China (No.2012CB921300) and the National Natural Science Foundation of China (Grant Nos.11274280 and 11104254). 


\section{References}

[1] Zhu F F, Chen W J, Xu Y, Gao C L, Guan D D, Liu C H, Qian D, Zhang S C and Jia J F 2015 Epitaxial growth of two-dimensional stanene, Nature Materials, 14.

[2] Vogt P, De P P, Quaresima C, Avila J, Frantzeskakis E, Asensio M C, Resta A, Ealet B and Le L G 2012 Silicene: compelling experimental evidence for graphenelike twodimensional silicon, Physical Review Letters, 108 489-92.

[3] Liu Z L, Wang M X, Xu J P, Ge J F, Lay G L, Vogt P, Qian D, Gao C L, Liu C and Jia J F 2014 Various atomic structures of monolayer silicene fabricated on $\operatorname{Ag}(111)$, New Journal of Physics, 16.

[4] Dávila M E, Xian L, Cahangirov S, Rubio A and Lay G L 2014 Germanene: a novel twodimensional Germanium allotrope akin to Graphene and Silicene, New Journal of Physics, 16 3579-87.

[5] Bianco E, Butler S, Jiang S, Restrepo O D, Windl W and Goldberger J E 2013 Stability and exfoliation of germanane: a germanium graphane analogue, Acs Nano, 7 4414-21.

[6] Jiang S, Butler S, Bianco E, Restrepo O D, Windl W and Goldberger J E 2014 Improving the stability and optical properties of germanane via one-step covalent methyltermination, Nature Communications, 5 163-80.

[7] Matusalem F, Marques M, Teles L K and Bechstedt F 2015 Stability and electronic structure of two-dimensional allotropes of group-IV materials, Physical Review B, 92.

[8] Matthes L, Pulci O and Bechstedt F 2013 Massive Dirac quasiparticles in the optical absorbance of graphene, silicene, germanene, and tinene, Journal of Physics Condensed Matter, 25395305.

[9] Xu Y, Gan Z and Zhang S C 2014 Enhanced thermoelectric performance and anomalous seebeck effects in topological insulators, Physical Review Letters, 112 770-7.

[10] Zhang G F, Li Y and Wu C 2014 Honeycomb lattice with multiorbital structure: Topological and quantum anomalous Hall insulators with large gaps, Physical Review $B, 90$.

[11] Wu S, Shan G and Yan B 2014 Prediction of Near-Room-Temperature Quantum Anomalous Hall Effect on Honeycomb Materials, Physical Review Letters, 113256401.

[12] Iijima S 1991 Helical microtubules of graphic carbon. 
[13] Woodside M T and Mceuen P L 2002 Scanned Probe Imaging of Single-Electron Charge States in Nanotube Quantum Dots, Science, 296 1098-101.

[14] Martel R, Schmidt T, Shea H R and Hertel T 1998 Single- and Multi-Wall Carbon Nanotube Field-Effect Transistors, Applied Physics Letters, 73 2447-9.

[15] Lee J O, Oh H, Kim J R, Kang K, Kim J J, Kim J and Yoo K H 2001 Nonlinear transport properties in multiwall carbon nanotube heterojunctions, Applied Physics Letters, 79 $1351-3$

[16] Postma H W, Teepen T, Yao Z, Grifoni M and Dekker C 2001 Carbon nanotube singleelectron transistors at room temperature, Science, 293 76-9.

[17] Heer W A D, Bonard J M and Stöckli T 1997 Carbon nanotubes films: electronic properties and their application as field emitters, Zeitschrift für Physik D Atoms, Molecules and Clusters, 40 418-20.

[18] Snow E S, Perkins F K, Houser E J, Badescu S C and Reinecke T L 2005 Chemical detection with a single-walled carbon nanotube capacitor, Science, 307 1942-5.

[19] Fagan S B, Baierle R J, Mota R, Silva A N J R D and Fazzio A 2000 Ab initio calculations for a hypothetical material: Silicon nanotubes, Physical Review B, 61 9994-6.

[20] Seifert G, K?Hler T, Urbassek H M, Hernández E and Frauenheim T 2001 Tubular structures of silicon, Physical Review B, 63 797-801.

[21] Yang X and Ni J 2005 Electronic properties of single-walled silicon nanotubes compared to carbon nanotubes, Physical Review B, 72.

[22] Saito R, Fujita M, Dresselhaus G and Dresselhaus M S 1992 Electronic structure of chiral graphene tubules, Applied Physics Letters, 60 2204-6.

[23] Hamada N, Si S and Oshiyama A 1992 New one-dimensional conductors: Graphitic microtubules, Physical Review Letters, 68 1579-81.

[24] Mintmire J W and White C T 1998 Universal Density of States for Carbon Nanotubes, Physical Review Letters, 81 2506-9.

[25] Cahangirov S, Topsakal M, Aktürk E, Sahin H and Ciraci S 2009 Two- and OneDimensional Honeycomb Structures of Silicon and Germanium, Physical Review Letters, 102236804.

[26] Xu Y, Yan B, Zhang H J, Wang J, Xu G, Tang P, Duan S C, and Zhang S C 2013 Large- 
gap quantum spin Hall insulators in tin films. Physical review letters, 111136804.

[27] Xiong W, Xia C, Peng Y, Du J, Wang T, Zhang J and Jia Y 2016 Spin-orbit coupling effects on electronic structures in stanene nanoribbons, Physical Chemistry Chemical Physics, 18 6534-40.

[28] Kresse G and Furthmüller J 1996 Efficient iterative schemes for ab initio total-energy calculations using a plane-wave basis set, Physical Review B Condensed Matter, 54 11169-86.

[29] Kresse G and Furthmüller J 1996 Efficiency of ab-initio total energy calculations for metals and semiconductors using a plane-wave basis set, Computational Materials Science, $\mathbf{6}$ $15-50$

[30] Kresse G and Joubert D 1999 From ultrasoft pseudopotentials to the projector augmentedwave method, Physical Review B, 59 1758-75.

[31] Perdew J P, Burke K and Ernzerhof M 1996 Generalized Gradient Approximation Made Simple, Physical Review Letters, 77 3865-8.

[32] Monkhorst H J and Pack J D 1976 Special points for Brillouin-zone integrations, Physical Review B, 13 5188--92.

[33] Tkatchenko A and Scheffler M 2009 Accurate molecular van der Waals interactions from ground-state electron density and free-atom reference data, Phys Rev Lett, 102073005.

[34] Pantea D, Brochu S, Thiboutot S, Ampleman G. \& Schol, G 2006 A morphological investigation of soot produced by the detonation of munitions. Chemosphere 65, 821831.

[35] Zhang R, Lee H-L, Li W-K and Teo B K 2005 Investigation of possible structures of silicon nanotubes via density-functional tight-binding molecular dynamics simulations and ab initio calculations, The Journal of Physical Chemistry B, 109 8605-12

[36] Ouyang M, Huang J L, Cheung C L and Lieber C M 2001 Energy gaps in "metallic" singlewalled carbon nanotubes, Science, 292 702-5.

[37] Kane C L and Mele E J 1997 Size, Shape and Low Energy Electronic Structure of Carbon Nanotubes, Physics, 78 1932-5.

[38] Blase X and Benedict L X 1994 Hybridization effects and metallicity in small radius carbon nanotubes, Physical Review Letters, 72 1878-81. 
[39] Heyd J and Ernzerhof M 2003 Hybrid functionals based on a screened Coulomb potential, Journal of Chemical Physics, 118 8207-15.

[40] Heyd J and Ernzerhof M 2006 Erratum: "Hybrid functionals based on a screened Coulomb potential” [J. Chem. Phys.118, 8207 (2003)], Journal of Chemical Physics, 124 219906--1. 